\title{
A Public Broadband Wireless Communication System on VHF TV Band
}

\author{
Hiroshi Harada, Masayuki Oodo, Ryuhei Funada \\ NICT \\ Yokosuka, Japan \\ E-mail:\{harada, moodo, funada\}@nict.go.jp
}

\author{
Mingtuo Zhou, Liru Lu, Xin Zhang \\ NICT Singapore Wireless Communications Laboratory \\ Singapore, Singapore \\ E-mail: \{mingtuo, liru, zhangxin\}@nict.com.sg
}

\begin{abstract}
This paper presents research status on public broadband wireless communication (PBB) system. The PBB system uses VHF band between $170 \mathrm{MHz}$ and $205 \mathrm{MHz}$ that is available after termination of analogue $\mathrm{TV}$ broadcasting service on July 24, 2011. This paper summarizes the transition of television service from analogue to digital and fundamental specification on spectrum usage, physical and MAC layers.
\end{abstract}

Keywords-component; $V H F$, Broadband, mobile, analogue TV, digital TV

\section{INTRODUCTION}

For the effective use of spectrum, current terrestrial analogue TV broadcasting service that uses $370 \mathrm{MHz}$ in total on VHF and UHF bands will be terminated on July 24, 2011 and only digital terrestrial TV in the UHF band from 470 to $710 \mathrm{MHz}$ will be available as shown in Fig. 1. By the transition to digital terrestrial TV, spectrum of $130 \mathrm{MHz}$ will be newly used for other use cases. The policy for the spectrum allocation of the vacant frequency band had been discussed and was decided as shown in Fig. 1. In the new frequency allocation plan, the most important use case is for the application to public broadband wireless communication (PBB) systems. Currently PBB users who work for police and fire department services and so on can only use voice and narrow band data communications. In the PBB systems, 35 $\mathrm{MHz}$ can be used in total between $170 \mathrm{MHz}$ and $205 \mathrm{MHz}$ and several applications based on video and high-resolution photograph are expected. To standardize the PBB system on VHF band, we have done a lot of research regarding use cases, system requirement, measurement and modeling on propagation characteristics, design of physical (PHY) and MAC layers, prototyping, and co-existing mechanism with adjacent wireless communication systems. This paper summarizes our research progress on the use case, the system requirement, the models of propagation characteristic, the design of physical (PHY) and MAC layers, and prospect of prototyping.

\section{USECASE AND SYSTEM REQUIREMENT}

Fig. 2 shows use cases of PBB system categorized into four: (a) between fixed base station (BS) and mobile stations (MSs), (b) between portable BS and MSs, (c) between MSs, (d) radio-relay. To realize the use cases, PBB systems need to consider some requirements. The first requirement is to secure communication zone as wide as possible, because PBB systems use VHF band with ten times lower frequency than microwave band and its transmission range expects to be lengthened.

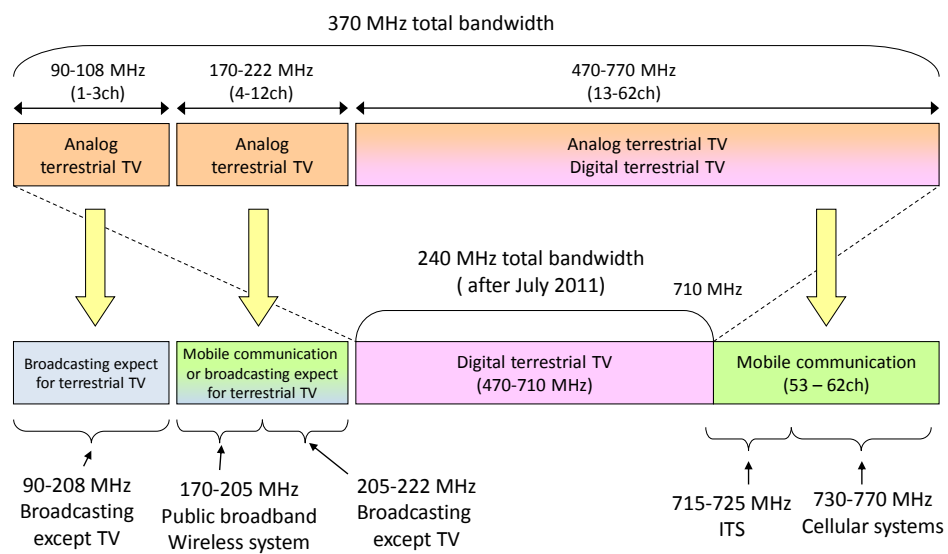

Fig. 1 Frequency allocation plan of VHF and UHF bands.

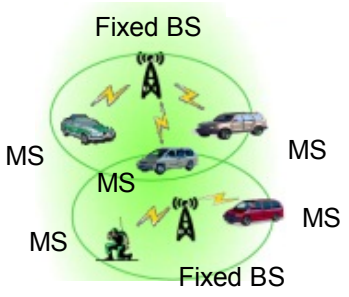

(a)

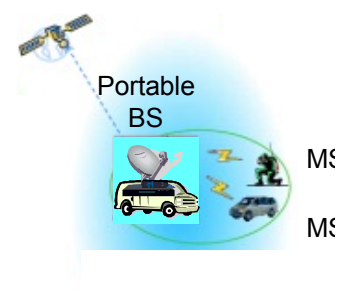

(b)

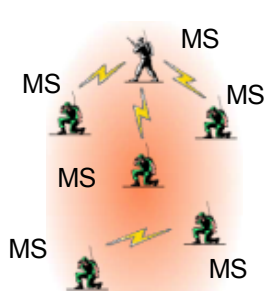

(c)

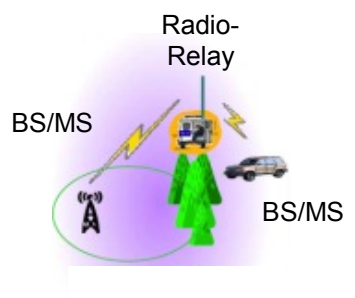

(d)
Fig. 2 Use cases of PBB system.

Second requirement is to support higher data throughput of uplink (UL) than down link (DL). This is because multimedia contents that include video and high resolution photograph of disaster area and crime scene must be sent to the commandservice place in the public safety situation. 
Third requirement is to support MSs as many as possible by a BS and the BS also needs to support several data rate generated from the MSs, respectively. Access schemes that can realize dynamic frequency and time slots access are required.

By considering the requirements, fundamental technical requirement regarding $\mathrm{PBB}$ was decided in the committee of Ministry and Internal Affairs and Communications (MIC) of Japan. The spectrum allocation plan for PBB system is shown in Fig. 3. Each channel has $5 \mathrm{MHz}$ and two $2.5 \mathrm{MHz}$ guard bands are allocated on the border with adjacent communication systems. Then, maximum transmission power is $20 \mathrm{~W}$ for fixed $\mathrm{BS}$ and $5 \mathrm{~W}$ for portable $\mathrm{BS}$ and MS. Regarding duplex scheme, time division duplex (TDD) is selected. This is because it is difficult to develop small-size bandpass filters for MS to secure uplink and downlink channel on VHF band in the case of frequency division duplex (FDD) and a large guard band may be needed between UL and DL channels.

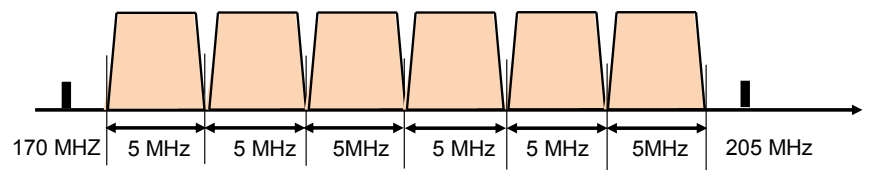

Fig. 3 Spectrum allocation plan for PBB system.

Regarding multiple access scheme, OFDMA is adopted because it is easy to realize dynamic frequency and time slots access by users and it has high connectivity with network. Moreover to realize video transmission that has equal or higher quality than NTSC, BPSK, QPSK, 16 QAM, and 64 QAM are selected as modulation scheme of each subcarrier in the OFDM. In addition, maximum antenna gain is $10 \mathrm{dBi}$ to minimize interference with the other wireless communication systems and broadcasting service. Based on the fundamental system requirement, $\mathrm{PHY}$ and MAC layers of PBB system are designed.

\section{MODELING OF PROPAGATION CHARACTERITICS}

To design PHY scheme for PBB system, it is essential to do performance analysis such as bit error rate (BER), packet error rate (PER) and so on by using radio propagation model specified to PBB. As already described, we have done radio propagation experiments in Numazu city by using measurement instruments that has $10 \mathrm{MHz}$ occupied bandwidth at centre frequency of $190 \mathrm{MHz}$ [1] and developed propagation models and developed three $\mathrm{PBB}$ radio new propagation models [2]. The difference between these three model is dependent on the range of zone, $d$. Table 1 summarizes the propagation models. The table also shows GSM typical urban model and a model used in IEEE 802.22 that considers regional area network (RAN) with transmission area of several tens $\mathrm{km}$ and that pursue to realize fix-to-fix communication. This table clearly indicates that delayed waves with the delay time of 10-20 us are received. So in the consideration of PHY and MAC scheme, more than 10-20 us of guard time is needed for OFDM design.
Table 1 Radio propagation model for PBB system.

\begin{tabular}{|c|l|c|c|c|c|c|c|}
\hline & & Path1 & Path2 & Path3 & Path4 & Path5 & Path6 \\
\hline \multirow{2}{*}{$\begin{array}{c}\text { PBB model 1 } \\
(\mathrm{d}<2.5 \mathrm{~km})\end{array}$} & Delay time [us] & 0 & 0.7 & 1.2 & 3.2 & 5.5 & 6.8 \\
\cline { 2 - 8 } & Relative power [dB] & 0 & -34.9 & -25.9 & -22.7 & -34.8 & -34.6 \\
\hline $\begin{array}{c}\text { GSM Typical } \\
\text { Urban }\end{array}$ & Delay time [us] & -0.2 & 0 & 0.3 & 1.4 & 2.1 & 4.8 \\
\cline { 2 - 8 } & Relative power [dB] & -3 & 0 & -2 & -6 & -8 & -10 \\
\hline $\begin{array}{c}\text { PBB model 2 } \\
(2.5 \mathrm{~km}<\mathrm{d}<5 \mathrm{~km})\end{array}$ & Delay time [us] & 0 & 0.9 & 1.7 & 3.1 & 3.8 & 7.5 \\
\cline { 2 - 8 } & Relative power [dB] & 0 & -18.2 & -20.6 & -25.0 & -26.5 & -19.6 \\
\hline $\begin{array}{c}\text { PBB model 3 } \\
(\mathrm{d}>5 \mathrm{~km})\end{array}$ & Delay time [us] & 0 & 0.6 & 5.3 & 6.2 & 7.5 & 19.5 \\
\cline { 2 - 8 } & Relative power [dB] & 0 & -12.1 & -25.2 & -22.2 & -18.5 & -21.8 \\
\hline $\begin{array}{c}\text { IEEE802.22 } \\
\text { Profile A model }\end{array}$ & Delay time [us] & 0 & 3 & 8 & 11 & 13 & 21 \\
\cline { 2 - 8 } & Relative power [dB] & 0 & -7 & -15 & -22 & -24 & -19 \\
\hline
\end{tabular}

\section{DESIGN OF PHY AND MAC LAYERS}

\section{A. Main direction}

PBB system must be based on OFDMA and TDD on VHF band. IEEE 802.22 system has almost same scope with the PBB system but the IEEE 802.22 system is for fix communication system. Some PHY parameters in the system, i.e. FFT size and so on, are quite high specification for the mobile use nevertheless some PHY parameters are optimized for the operation in VHF band. Moreover, IEEE802.16-2009 based wireless communication system supports wireless communication between BS and MSs. But mainly the operational frequency band is microwave band not VHF band. So even if the operational frequency band is changed to VHF, some PHY parameters of IEEE802.16-2009 may need to be changed. Based on the above discussion, as the first step, MIC, Japan entrusted ARIB, Japanese standardization body, to provide standard for $200 \mathrm{MHz}$-band wireless communication systems for the use case (a) in Fig. 2 that is a wireless communication system between portable BS and MSs. ARIB has standardized two PHY modes shown in Table 2. Mode 1 is completely based on IEEE 802.16-2009 and just changes its operational frequency band. Mode 2 is a modified version of mode 1 and optimized for requirements for PBB application.

\section{B. Mode 1}

Mode 1 is completely based on IEEE 802.16-2009 and just changes its operational frequency band. Mode 1 selected two types of FFT size, 512 and 1024 [2]. The reason to select two types is dependent on how large delayed waves need to be considered. If we design a small communication zone, maximum delay time of delayed wave is smaller than 10 us from Table 1. In this case, 512 FFT size can be selected because the cyclic prefix length for guard interval in each OFDM symbol is 11.4 us. On the other hand, if we design a large communication zone, maximum delay time of delayed wave is larger than 10 us, in this case 1024 FFT size is appropriate because the cyclic prefix length is 22.9 us. But even if 1024 FFT size is selected, mode 1 can not achieve $10^{-6}$ of BER performance in some situations. Fig. 4 shows BER performance when 16QAM-OFDM and convolutional code with constrain length $\mathrm{K}=7$ and coding rate $\mathrm{R}=1 / 2$ is transmitted under the PBB model 3 channel in Table 1 . In this case, UL transmission achieves $10^{-6}$ of BER performance but DL can not achieve the performance. The reason is dependent on pilot symbol assignment. Fig. 5 shows the pilot symbol assignment when 1024 FFT size is selected. The duration of 
Table 2 PHY and MAC parameters for PBB system.

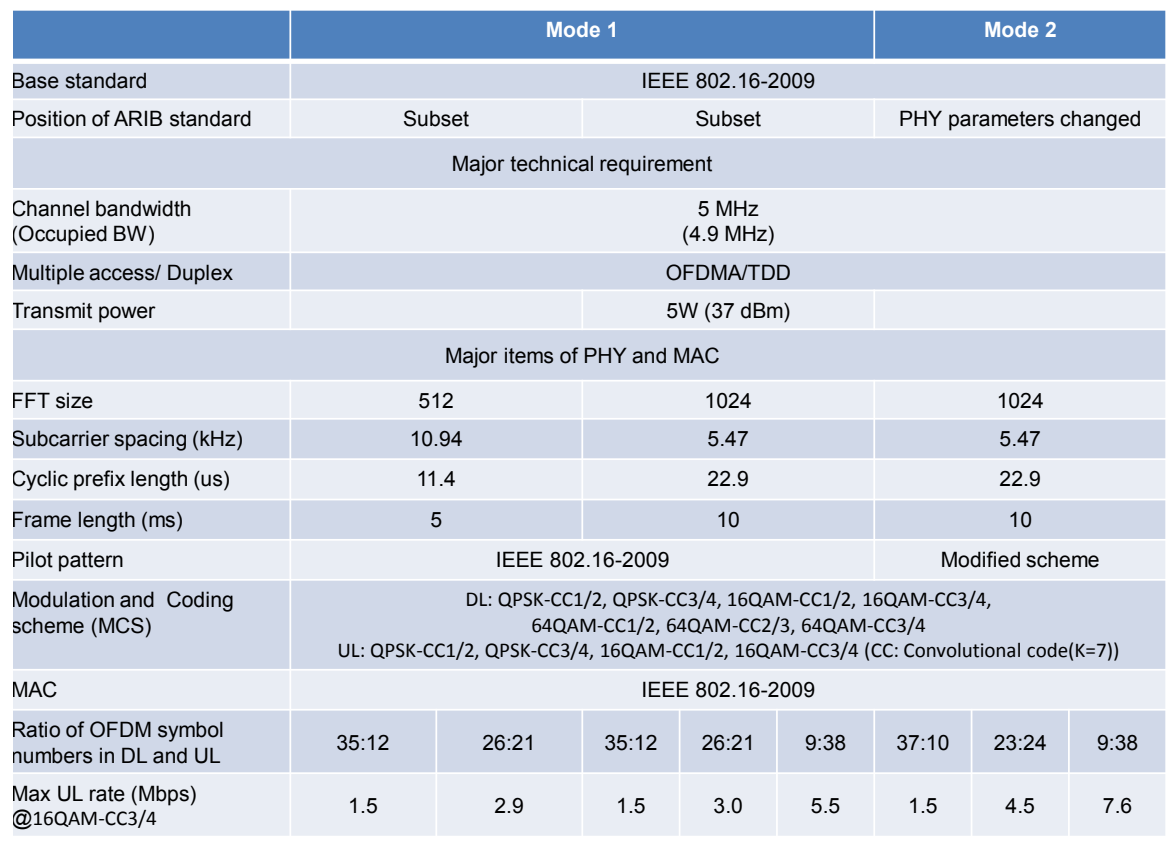

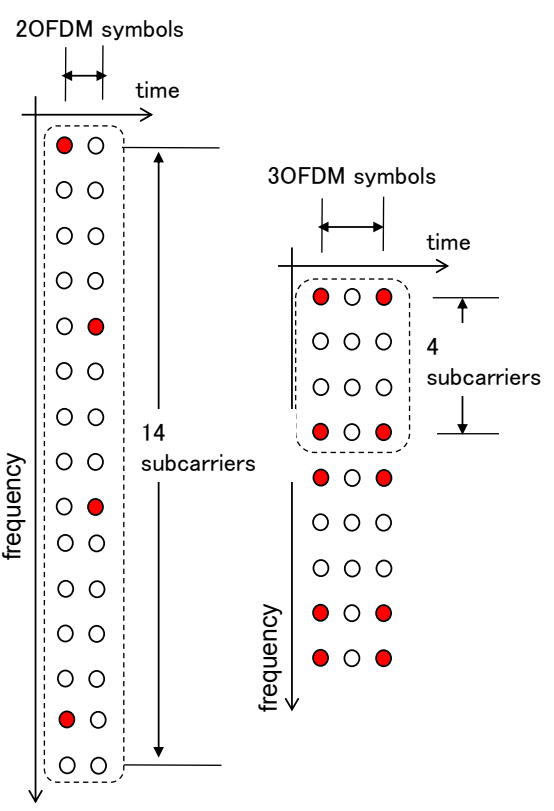

DL

UL

Fig. 5 Pilot symbol assignment for mode 1 (FFT size: 1024).

pilot symbol insertion in frequency domain for DL is quite larger than that for UL. So, in some cases, the pilot symbol inserted in DL may not be able to estimate propagation channel accurately. So, in order to realize better DL BER performance under $\mathrm{PBB}$ channel model, the pilot symbol insertion in DL scheme must be modified. Moreover, from Table 2, maximum transmission rate of UL in mode 1 is at most 5.5 Mbps in the case using 16QAM-OFDM. To realize high definition video transmission, at least $7 \mathrm{Mbps}$ transmission rate is needed for UL. So in order to realize such transmission rate, we may reduce the number of pilot symbols in UL. But in this case, we may also reduce the performance of channel estimation in multipath fading channel.

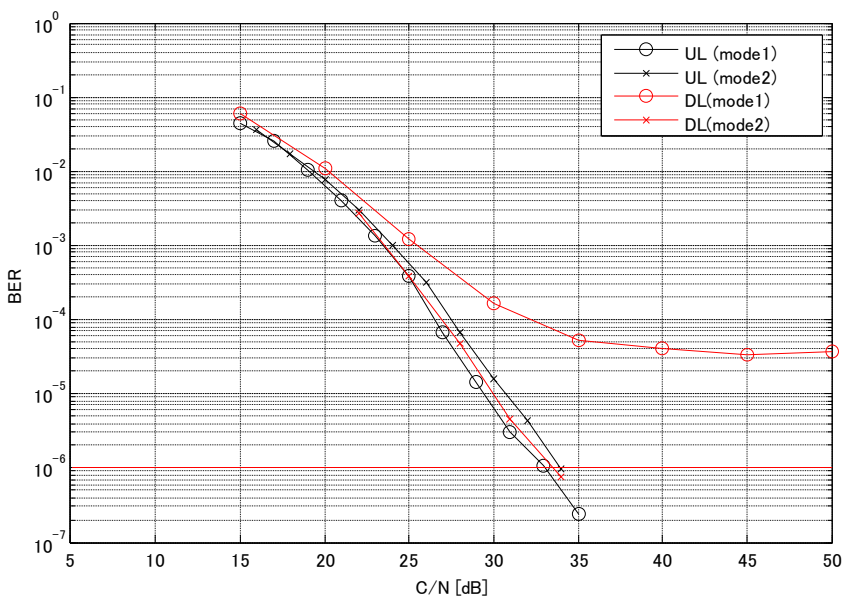

Fig. 4 BER performance when 16QAM-OFDM is used.

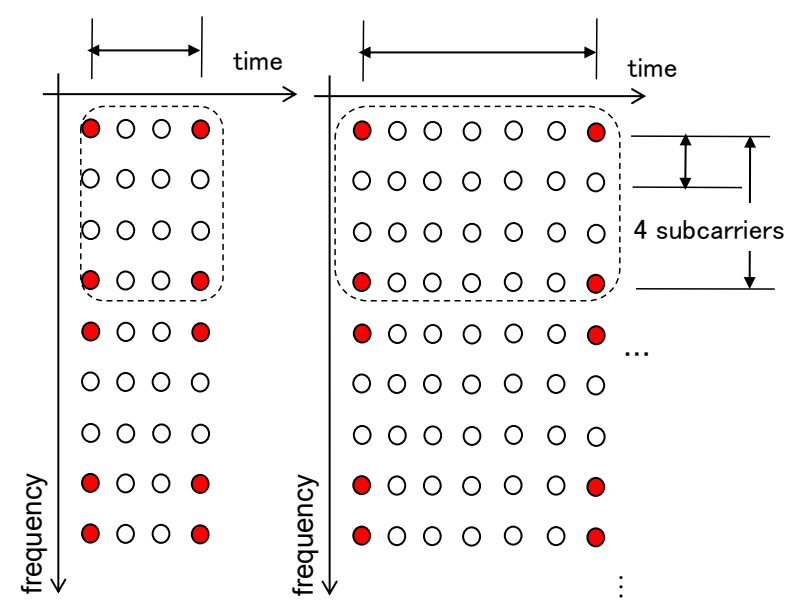

Fig. 6 Pilot symbol assignment for mode 2 (FFT size: 1024).

\section{Mode 2}

Mode 2 is a modified version of mode 1 . Major change is to change pattern of pilot symbol insertion for both UL and DL. Fig. 6 shows the pattern used in mode 2. For DL, the interval of pilot symbol insertion is reduced in comparison with mode 1 . This means that DL of mode 2 is much robust against multipath fading in comparison with mode 1. Fig. 4 shows the performance of mode 2. The BER performance in both DL and UL achieves $10^{-6}$. On the other hand, for UL, the interval of pilot symbol insertion is increased in comparison with mode 2 . The insertion interval in mode 1 is good for operational frequency band for IEEE 802.16 such as $2.5 \mathrm{GHz}$ and so on. The operational frequency of PBB system is around $200 \mathrm{MHz}$. So even if the interval of pilot symbol insertion is increased in comparison with mode 2, the BER performance seems not to be degraded. 
Table 3 Required CNR to achieve $10^{-6}$ of BER performance when 16 QAM-OFDM and convolutional code with $\mathrm{K}=7$ and $\mathrm{R}=1 / 2$ is used.

\begin{tabular}{|c|c|c|c|c|c|c|c|}
\hline \multirow[t]{2}{*}{ Mode } & \multirow[t]{2}{*}{ Link } & \multirow{2}{*}{$\begin{array}{l}\text { Modulation and } \\
\text { Coding Scheme } \\
\text { (MCS) }\end{array}$} & \multicolumn{5}{|c|}{ Required CNR to achieve $10^{-6}$} \\
\hline & & & $\begin{array}{c}\text { PBB } \\
\text { Model } 1\end{array}$ & $\begin{array}{l}\text { GSM Typical } \\
\text { Urban Model }\end{array}$ & $\begin{array}{c}\text { PBB } \\
\text { Model } 2\end{array}$ & $\begin{array}{c}\text { PBB } \\
\text { Model } 3\end{array}$ & $\begin{array}{c}\text { IEEE } 802.22 \\
\text { Profile A Model }\end{array}$ \\
\hline \multirow{4}{*}{$\begin{array}{l}\text { Mode } \\
1 \text { (FFT } \\
\text { size=1 } \\
024)\end{array}$} & \multirow[b]{2}{*}{ UL } & QPSK-CC1/2 & $32 \mathrm{~dB}$ & $17 \mathrm{~dB}$ & $26 \mathrm{~dB}$ & $25 \mathrm{~dB}$ & $24 \mathrm{~dB}$ \\
\hline & & 16QAM-CC1/2 & $37 \mathrm{~dB}$ & $\begin{array}{r}23 \mathrm{~dB} \\
(33 \mathrm{~dB}, 16 \mathrm{1} A \mathrm{M}- \\
\mathrm{CC} / 4)\end{array}$ & $32 \mathrm{~dB}$ & $33 \mathrm{~dB}$ & $33 \mathrm{~dB}$ \\
\hline & \multirow{2}{*}{$\mathrm{DL}$} & QPSK-CC1/2 & $35 \mathrm{~dB}$ & $19 \mathrm{~dB}$ & $28 \mathrm{~dB}$ & $29 \mathrm{~dB}$ & N/A \\
\hline & & 16QAM-CC1/2 & $45 \mathrm{~dB}$ & $27 \mathrm{~dB}$ & $35 \mathrm{~dB}$ & N/A & N/A \\
\hline \multirow{4}{*}{$\begin{array}{l}\text { Mode } \\
2\end{array}$} & \multirow{2}{*}{ UL } & QPSK-CC1/2 & $32 \mathrm{~dB}$ & $18 \mathrm{~dB}$ & $27 \mathrm{~dB}$ & $25 \mathrm{~dB}$ & $24 \mathrm{~dB}$ \\
\hline & & 16QAM-CC1/2 & $38 \mathrm{~dB}$ & $26 \mathrm{~dB}$ & $34 \mathrm{~dB}$ & $34 \mathrm{~dB}$ & $37 \mathrm{~dB}$ \\
\hline & \multirow{2}{*}{$\mathrm{DL}$} & QPSK-CC1/2 & $32 \mathrm{~dB}$ & $17 \mathrm{~dB}$ & $26 \mathrm{~dB}$ & $25 \mathrm{~dB}$ & $25 \mathrm{~dB}$ \\
\hline & & 16QAM-CC1/2 & $37 \mathrm{~dB}$ & $23 \mathrm{~dB}$ & $32 \mathrm{~dB}$ & $33 \mathrm{~dB}$ & $35 \mathrm{~dB}$ \\
\hline
\end{tabular}

Fig. 4 also shows that the BER performances between mode 1 and mode 2 are almost same. However, if the interval of pilot symbol insertion is increased, more data symbols can be transmitted. In the case of mode 2 and 9:38 of DL and UL ratio, 7.6 Mbps can be achieved. This rate is appropriate to transmit high definition video contents. Table 3 shows the required CNR to achieve $10^{-6}$ of BER performance when 16 QAM-OFDM and convolutional code with $\mathrm{K}=7$ and $\mathrm{R}=1 / 2$ is used for all modes in all PBB channel models. From the table, mode 2 can achieve $10^{-6}$ of BER performance in all PBB channel model.

\section{Prototype}

Based on the design of PHY and MAC layers for modes 1 and 2, a prototype is developing. Table 4 shows fundamental parameter of the prototype and Fig. 7 shows a photograph of the prototype. We recognize that mode 1 and 2 can be feasible for the implementation.

Table 4 Specification of prototype.

\begin{tabular}{|l|l|}
\hline item & Specification \\
\hline $\begin{array}{l}\text { Center frequency } \\
\text { band }\end{array}$ & $175 \mathrm{MHz} \sim 200.0 \mathrm{MHz}$ \\
\hline $\begin{array}{l}\text { Transmission power } \\
\text { Bandwidth }\end{array}$ & $5 \mathrm{~W}$ \\
\hline IF frequency band & $5 \mathrm{MHz}$ \\
\hline FPGA & $20 \mathrm{MHz}$ \\
\hline Memory & Xilinx XC5VLX330,XC5VLX220 \\
\hline Battery life time & SDRAM 256 Mbps \\
\hline & 1.5 hours (continuous transmission mode) \\
\hline
\end{tabular}

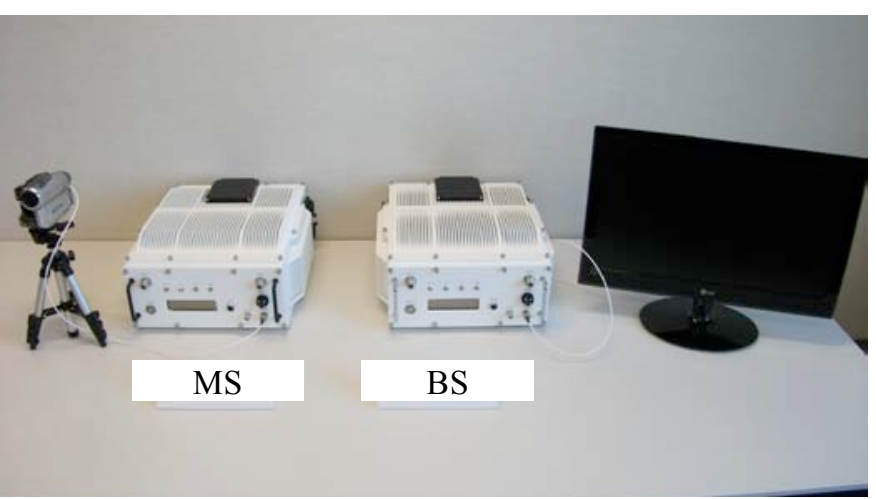

Fig. 7 Photograph of prototype.

\section{CONCLUSION}

This paper summarized use case, the system requirement, the models of propagation characteristic, the design of PHY and MAC layers and finally prospect of prototyping regarding PBB systems used VHF band. The PBB system includes two modes and especially mode 2 is optimized to PBB system and PBB operational frequency band. By using the PBB mode 2 system, transmission rate that realizes high definition video contents transmission is achieved. As a further work, we will do field test by using the developed prototype.

\section{REFERENCES}

[1] M. Oodo, N. Soma, and H. Harada, "Radio propagation experiments for broadband wireless communication system in the VHF band," Proc. WPMC 2008, Sept. 2008.

[2] IEEE Std 802.16TM-2009, IEEE standard for local and metropolitan area networks Part 16: Air Interface for Broadband Wireless Access Systems. 